\title{
Oral Administration of Fucoxanthin Increases Plasma Fucoxanthinol Concentration and Antioxidative Ability and Improves Meat Color in Broiler Chicks
}

\author{
Keisuke Sasaki ${ }^{1}$, Kenji Ishihara ${ }^{2}$, Makoto Yamazaki ${ }^{1}$, Kazuki Nakashima ${ }^{1}$, Hiroyuki Abe ${ }^{1}$, \\ Chiaki Oyamada ${ }^{2}$, Michiyo Motoyama $^{1}$ and Mitsuru Mitsumoto ${ }^{1, a}$ \\ ${ }^{1}$ National Institute of Livestock and Grassland Science, 2 Ikenodai, Tsukuba, Ibaraki 305-0901, Japan \\ ${ }^{2}$ National Research Institute of Fisheries Science, 2-12-4 Fukuura, Kanazawa-ku, Yokohama, Kanagawa 236-8648, Japan
}

\begin{abstract}
Effects of the oral administration of fucoxanthin (FX), a major carotenoid of algae, on plasma fucoxanthinol (FX-OH) concentration, plasma antioxidative status, and meat color and lipid stability during chilling storage in broiler chicks were investigated. One-week-old male broiler chicks were divided into control and FX treatment groups. For the FX treatement group, FX was given into the crop of each bird at a volume of $10 \mathrm{mg}$ per day per bird for 2 weeks. After 2 weeks of treatment, the plasma, liver, and superficial pectoral muscle were harvested. FX was not detected in either the plasma or liver of control and FX-treated birds, although FX-OH was detected in both the plasma and liver of the FX-treated group. Plasma antioxidative activity against a peroxyl radical derived from 2, 2' -azobis-(2-amidinopropane) dihydrochloride was observed in FX-treated chicks but not in control chicks. FX treatment did not affect the $L^{*}$ and $a^{*}$ values nor the lipid peroxide content of superficial pectoral muscle during 6 days of chilling storage. However, FX treatment increased the $b^{*}$ value of superficial pectoral muscle compared to the control group $(P<0.05)$. These results indicated that FX was incorporated and converted into $\mathrm{FX}-\mathrm{OH}$ during absorption, and that $\mathrm{FX}-\mathrm{OH}$ acted as an antioxidant in chick plasma. It was also revealed that the FX treatment for broiler chicks affected their meat color. Our results suggested that FX is a potent antioxidative feed ingredient for enhancing both the plasma antioxidative status and meat color in broiler chicks.
\end{abstract}

Key words: antioxidative status, broiler chicks, feed additive,fucoxanthin, meat color

J. Poult. Sci., 47: 316-320, 2010

\section{Introduction}

Antioxidative molecules in chickens are important factors in maintaining productivity and hygiene. For example, dietary vitamins $\mathrm{C}$ and $\mathrm{E}$ were found to improve antioxidant status, immune responses and production performance of White Leghorn layers under summer conditions (Panda et al., 2008). Dietary ascorbic acid also acted as an antioxidant against radical initiator- or heat stress-derived oxidative stresses (Sasaki et al., 2002a; Aoyagi et al., 1996; Aoyagi et al., 1997). Dietary antioxidants have been also investigated for the improvement of meat quality. For example, there have been various reports of dietary vitamin E maintaining the lipid and color stability of chicken meat as summarized by Mitsumoto (2000).

Received: March 3, 2010, Accepted: August 6, 2010

Released Online Advance Publication: September 25, 2010

Correspondence: Dr. K. Sasaki, National Institute of Livestock and Grassland Science, 2 Ikenodai, Tsukuba, Ibaraki 305-0901, Japan.

(E-mail: ksuk@affrc.go.jp)

${ }^{a}$ Deceased on $28 \mathrm{Feb} 2007$
Moreover, the applications of useful and effective natural antioxidants have been investigated for animal and poultry production. Carotenoids have the potential to serve as useful natural antioxidants, e.g., Mortensen and Skibsted (2000) have reviewed several studies for the possible application of dietary carotenoids to improve chicken products. Since these reports appeared in studies of $\beta$-carotene and astaxanthin, other molecular species of carotenoids should be examined as possible antioxidants in poultry production.

Fucoxanthin (FX), (3S, 5R, 6S, 3'S, 5'R, 6' R)-3'acetoxy-5, 6-epoxy-3, 5'-dihydroxy-6' , $^{\prime}$-didehydro-5, 6, 7, $8,5^{\prime}, 6^{\prime}$-hexahydro- $\beta, \beta$-caroten- 8 -one (Figure 1 ), is a major carotenoid in Phaeophyceae (algae), and is the most biosynthesized carotenoid worldwide (Haugan et al., 1992). FX has proton-donative antioxidative activity (Nomura et al., 1997) and is metabolized into fucoxanthinol (FX-OH) in the intestinal tract during absorption (Sugawara et al., 2002). FX-OH also acts as the antioxidant in animal plasma (Sachindra et al., 2007). Based on these findings, FX is expected to prove a useful antioxidative feed additive for maintaining antioxidative status in chicks. In the pre- 


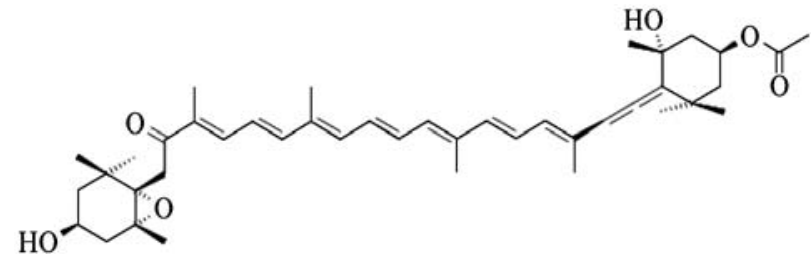

Fig. 1. Structure of fucoxanthin

sent study, to evaluate the possibility of FX as an effective feed additive for maintaining antioxidative status in chickens and other fowl we investigated the effects of oral FX administration on plasma $\mathrm{FX}-\mathrm{OH}$ concentration and radical trapping activity in broiler chicks.

In addition, we previously reported that postmortem FX supplementation to chicken breast meat adversely affected meat color and prevented lipid peroxidation during chilling storage after cooking treatment (Sasaki et al., 2008). Therefore, another aim of the present study is to investigate the effects of oral FX treatment on the color and lipid stability of postmortem chicken meat during chilling storage.

\section{Materials and Methods}

\section{FX Preparation}

FX was prepared from the Japanese edible seaweed Wakame (Undaria pinnatifida), as described previously (Haugan et al., 1992). Briefly, acetone-methanol (70:30, $\mathrm{v} / \mathrm{v}$ ) extraction of dried Wakame samples was performed, and the partition of hexane from aqueous methanol was conducted to remove chlorophylls and less polar carotenoids. The extract was subjected to silica-gel column chromatography for FX purification, as described by Sugawara et al. (2001). For column chromatography, hexane-ethyl acetate (100:0, 80:20, 60:40, 50:50, and 40:60, v/v) was used for elution. The eluent of hexane-ethyl acetate from 50:50 to 40:60 was collected as the FX fraction.

We estimated the purity of FX by spectrophotometric assay and high-performance liquid chromatography (HPLC). The HPLC assay was performed as described by Haugan et al. (1992) on a Develosil CN-UG-5 column $(250 \times 4.6$ $\mathrm{mm})$. FX was monitored with an ultraviolet/visible detector (No. 119 UV/VIS detector, Gilson, Inc., Middleton, WI) set at $445 \mathrm{~nm}$. The mobile phase was hexane-isopropyl acetate-acetone-methanol (76:17:7:0.1, v/v/v/v), and the flow rate was $2.0 \mathrm{ml} / \mathrm{min}$. The purity of $\mathrm{FX}$ used in the present study was estimated as $94.2 \%$. HPLC analysis showed that FX was composed of $95.9 \%$ all-trans-FX and $4.1 \%$ cis-FX.

\section{Animals}

One-week-old male broiler chicks were obtained from Nippon Chunky Co., Ltd. (Okayama, Japan). Fourteen birds were divided into two groups, one the control and the other the FX group. Initial body weight of each group was $201.4 \pm 7.3 \mathrm{~g}$ for control group and $198.0 \pm 7.7 \mathrm{~g}$ for FX group (mean $\pm S D$ ). Each bird was housed in an in- dividual cage, and applied to FX administration testings for two weeks. During the experiment, the chicks were fed a corn-soybean based commercial starter diet (crumble form, $3.2 \mathrm{kcal} / \mathrm{g} \mathrm{ME}, 22 \% \mathrm{CP}$; Sugiji feed mills, Aichi, Japan) ad libitum. All nutrient contents met or exceeded the National Research Council recommendation (National Research Council, 1994). Light was provided continuously, and ambient temepature was maintained at $30^{\circ} \mathrm{C}$.

All birds received humane care as outlined in the Guide for the Care and Use of Experimental Animals (Animal Care Committee, National Institute of Livestock and Grassland Science).

\section{FX Administration Experiments}

FX was dissolved in ethanol and suspended in a fourthvolume of corn oil. The FX suspension was then emulsified into a tenth-volume of $0.2 \%$ xanthan gum solution. This emulsion was used as an FX sample for oral administration, the final concentration of FX was $5 \mathrm{mg} / \mathrm{mL}$. FX emulsion was given into the crop of each bird at a volume of $2 \mathrm{~mL}$ per day, yielding a daily FX intake of $10 \mathrm{mg}$ in each chick. FX administration continued at 13:00 of every day for 14 days. For the control group, FX-free emulsion was prepared and injected similar to that in the FX administration group. On the final day of FX treatment, the birds were killed by anesthesia and bleeding, and blood, liver, and superficial pectoral muscle were harvested. Plasma was immediately separated from blood, and used to assay radical trapping activity and $\mathrm{FX}$ and $\mathrm{FX}-\mathrm{OH}$ determination plasma samples of which were stored at $-80^{\circ} \mathrm{C}$ until analysis.

\section{Chemical Determination and Radical Trapping Activity}

FX and FX-OH were extracted from plasma and liver with a chloroform-methanol (2:1) reagent including 0.05 $\%$ butylated hydroxytoluene, and were determined by a HPLC method as described above.

For the investigation of plasma radical trapping activity, plasma lipid peroxide was determined before and after treatment of the radical initiator. As for lipid peroxide, thiobarbituric reactive substances (TBARS) were determined by spectrophotometric assay as described previously (Sasaki et al., 2002a). In the treatment with a radical initiator, 2,2'-azobis-2-amidinopropane dihydrochloride (AAPH) was used. AAPH has been reported to be a useful peroxyl radical initiator (Niki, 1990), and AAPH injection has been used as a model for radical injury in chicks (Sasaki et al., 2002a; Sasaki et al., 2002b; Aoyagi et al., 1996). In the present study, an equal volume of 100 $\mu \mathrm{M}$ AAPH solution was added to plasma and incubated at $37^{\circ} \mathrm{C}$ for 30 minutes for the treatment of radical injury. The final AAPH concentration was $50 \mu \mathrm{M}$, which is effective concentration in vivo investigation (Sasaki et al., 2002 a).

\section{Meat Storage Experiment and Measurement}

Muscle samples were minced and formed into circular patties $35 \mathrm{~mm}$ in diameter and $10 \mathrm{~mm}$ in thickness. The patties were put into a weighing boat, over-wrapped by oxygen-permeable PVC film, and then stored in a refriger- 
ator set at $4^{\circ} \mathrm{C}$ in the dark or under fluorescent light for 1 day or 6 days. Meat color parameters such as $a^{*}, b^{*}$, and $L *$ values were analyzed by a UV-2400PC spectrophotometer (Shimadzu Co., Tokyo) equipped with an ISR-2200 integrating sphere (Shimadzu Co., Tokyo). Lipid peroxide was evaluated based on the TBARS values, which were determined by spectrophotometric assay as described (Witte et al., 1970) and later modified (Mitsumoto et al., 1993), with the values were expressed as nmol malondialdehyde equivalents per g samples.

\section{Statistical Analysis}

Comparisons of plasma TBARS values between before and after AAPH treatment were analyzed by paired $t$-test. Daily body weight gain, feed intake, feed efficiency, liver and superficial pectoral muscle weight, meat color parameters, and TBARS values were analyzed by the general liner model procedure of SAS (version 9.12, SAS Institute, Cary, NC).

\section{Results and Discussion}

According to a previous study on mice and Caco- 2 cell line established from human colonic carcinoma, FX is metabolized into $\mathrm{FX}-\mathrm{OH}$ in the intestinal tract during absorption (Sugawara et al., 2002). Accordingly, we determined both FX and $\mathrm{FX}-\mathrm{OH}$ in the plasma of chicks orally administered FX. No FX was detected by the HPLC method in either plasma or liver in both the control and FX-administrated groups (data not shown). On the other hand, FX-OH was detected in plasma and liver in FXtreated chicks, but not in control chicks as presented in Table 1. From these results, we considered that FX was converted into $\mathrm{FX}-\mathrm{OH}$ during absorption in a way similar to that in mammals.

Table 2 presents the daily body weight gain, feed intake,

Table 1. Liver and plasma fucoxanthinol concentration

\begin{tabular}{lcc}
\hline \hline & \multicolumn{2}{c}{ Group } \\
\cline { 2 - 3 } & Control & Fucoxanthin treated \\
\hline Liver $(\mathrm{nmol} / \mathrm{g})$ & N.D. & $1.35 \pm 0.98$ \\
Plasma $(\mu \mathrm{M})$ & N.D. & $0.58 \pm 1.13$ \\
\hline
\end{tabular}

Values are expressed as mean $\pm \mathrm{SD}$.

N.D.: Not detectable. feed efficiency, and liver and superficial pectoral muscle weight of the control and FX-treated groups. FX treatment did not affect the daily body weight gain, feed intake, feed efficiency, and liver weight $(P>0.05)$. The weight of superficial pectoral muscle, however, was significantly lower in the FX-treated than the control group $(P<0.05)$. Our results indicated that oral FX injections did not affect the growth performance of broiler chicks but did decrease their muscle yield. These results suggested that oral FX administration affects body composition in broiler chicks. The body composition, especially fat mass, in FX treated chicks should be investigated in further.

Figure 2 shows plasma TBARS values before and after the AAPH treatment. Although in the control group, plasma TBARS values increased by the AAPH treatment $(P<0.05)$, they remained unchanged by AAPH treatment in FX-treated chick plasma. This result demonstrates that the plasma of FX-treated chicks has a stronger defensive ability against radical molecules derived from AAPH than that of control chicks. In addition, $\mathrm{FX}-\mathrm{OH}$ has also been known as an antioxidant, and its radical scavenging activity is similar to that of FX (Sachindra et al., 2007). From the results shown in Table 1 and Figure 2, we concluded that FX was incorporated and converted into $\mathrm{FX}-\mathrm{OH}$, which acted as an antioxidant in chick plasma.

Table 3 presents the effects of oral FX administration on

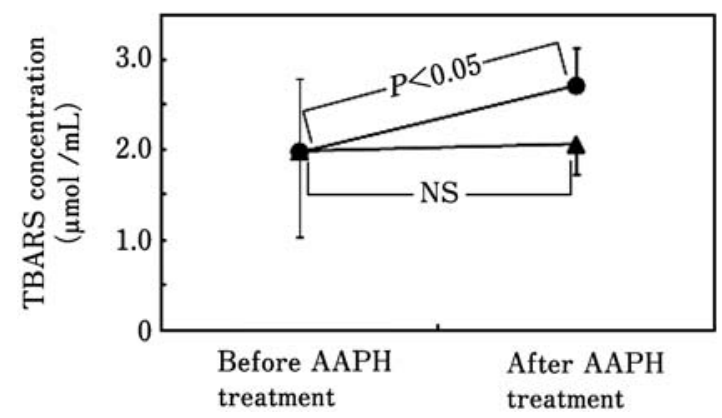

Fig. 2. Thibarbituric acid reactive substance (TBARS) concentration in control and fucoxanthin-treated chick plasma before and after treatment of radical initiator 2,2'-azobis(2-amidinopropane) dihydrochloride (AAPH). Values are expressed as means $\pm \mathrm{SD}$.

: Control group. $\boldsymbol{\Delta}$ : Fucoxanthin-treated group.

Table 2. Daily body weight gain, feed intake, feed efficiency, and liver and superficial pectoral muscle weight of control and fucoxanthin-treated group

\begin{tabular}{lccc}
\hline \hline & Control & Fucoxanthin treated & \\
\hline Body weight gain (g/day) & $55.56 \pm 4.57$ & $48.36 \pm 8.51$ & $\mathrm{~ns}$ \\
Feed intake (g/day) & $70.96 \pm 5.00$ & $63.99 \pm 10.39$ & $\mathrm{~ns}$ \\
Feed efficiency & $0.78 \pm 0.02$ & $0.75 \pm 0.04$ & $\mathrm{~ns}$ \\
Liver weight (g) & $22.66 \pm 3.67$ & $22.07 \pm 2.59$ & $\mathrm{~ns}$ \\
Superficial pectoral muscle weight (g) & $120.3 \pm 6.0$ & $103.1 \pm 17.5$ & $P<0.05$ \\
\hline
\end{tabular}

Values are expressed as means \pm SD.

ns: not significant $(P>0.05)$. 
Table 3. Effects of oral FX administration on meat color and thiobarbituric acid reactive substances (TBARS) in ground superficial pectoral muscle during storage in the dark or under fluorescent lightning

\begin{tabular}{|c|c|c|c|c|c|}
\hline & & \multicolumn{3}{|c|}{ Meat color } & \multirow{2}{*}{$\begin{array}{c}\text { TBARS } \\
(\mu \mathrm{mol} / \mathrm{g})\end{array}$} \\
\hline & & $L^{*}$ & $a^{*}$ & $b^{*}$ & \\
\hline \multirow[t]{4}{*}{ Treatment } & & ns & ns & $* * *$ & ns \\
\hline & Control & 49.0 & 5.3 & 14.0 & 1.41 \\
\hline & Fucoxanthin & 49.8 & 6.1 & 15.5 & 1.38 \\
\hline & SE & 0.3 & 0.4 & 0.2 & 0.20 \\
\hline \multirow{4}{*}{ Light condition } & & ns & $* *$ & $* *$ & ns \\
\hline & Dark & 49.5 & 5.5 & 14.6 & 1.53 \\
\hline & Light & 49.2 & 5.9 & 15.0 & 1.26 \\
\hline & SE & 0.2 & 0.1 & 0.1 & 0.12 \\
\hline \multirow[t]{4}{*}{ Storage days } & & $* * *$ & ns & ns & $* * *$ \\
\hline & Day 1 & 50.9 & 5.8 & 14.9 & 0.78 \\
\hline & Day 6 & 47.8 & 5.6 & 14.6 & 2.01 \\
\hline & SE & 0.2 & 0.1 & 0.1 & 0.12 \\
\hline \multicolumn{2}{|c|}{ Treatment $\times$ Light condition } & ns & ns & ns & ns \\
\hline \multicolumn{2}{|c|}{ Treatment $\times$ Storage days } & ns & ns & ns & ns \\
\hline \multicolumn{2}{|c|}{ Light condition $\times$ Storage days } & ns & ns & ns & $\mathrm{ns}$ \\
\hline \multicolumn{2}{|c|}{ Treatment $\times$ Light condition $\times$ Storage days } & ns & ns & * & ns \\
\hline
\end{tabular}

meat color and lipid stability in the superficial pectoral muscle during storage in the dark or under fluorescent lightning. Lighting condition did not affect either meat color parameters or TBARS values during storage $(P>$ $0.05)$. Meat color parameters such as $a^{*}$ and $L^{*}$ values did not change in the FX-treated group compared to the control group $(P>0.05)$, although $b^{*}$ values were higher in the former group $(P<0.05)$. In preliminary analysis, yellowness of intraperitoneal adipose tissue is also higher in FX-treated group than control group (data not shown). These results suggested that FX-OH may be incorporated into muscles and act as a pigment. TBARS values as presented in Table 3, however, were not affected by FX treatment. We previously reported that the postmortem FX supplementation to chicken breast meat prevented lipid peroxidation during storage after cooking, but not before (Sasaki et al., 2008). In this study, the storage experiment was conducted before cooking. The result that lipid peroxidation was not prevented in the meat of FX treated chicks may be attributable to the storage experiment before cooking. A study of the effect of oral FX treatment on the lipid stability of chicken meat after cooking is needed to evaluate the potential of FX as antioxidative feed additives to maintain lipid stability in chicken meat. In addtition, the results indicated in table 1 suggest that muscles of $\mathrm{FX}$-fed birds contain $\mathrm{FX}-\mathrm{OH}$, not FX, although FX was supplied to muscle samples in our previous study (Sasaki et al., 2008). Difference in antioxidative function between FX and FX-OH in muscle foods also should be investigated. On the other hand, our results indicated that the oral FX administration adversely affects the meat color of superficial pectoral muscle. We previously reported that postmortem FX supplementation to chicken breast meat increased $a^{*}$ and $b^{*}$ values but decreased the $L^{*}$ value (Sasaki et al., 2008). The disparity in results between the present and previous studies may be due to the differences between oral and exogenous additions of FX. Further studies will be needed into the effects of FX and FX-OH on meat color and lipid stability during storage both before and after cooking. In addition, meat color is an important characteristic in consumers' selection of meat. Consumers' impression of FX-treated meat color also should be investigated for establishment of FX as feed additives.

In conclusion, the present study indicated that the oral administration of FX increased plasma FX-OH and antioxidative activity in broiler chicks. Moreover, oral FX supplementation also increased the $b^{*}$ value of chicken superficial pectoral muscle without affecting the lipid stability of the muscle during chilling storage. These findings suggested that FX or FX-rich algae are potentially useful feed ingredients for maintaining the antioxidative status of chicks and improving chicken meat color.

\section{Acknowledgments}

This work was supported in part by the Fisheries Agency of the Ministry of Agriculture, Forestry, and Fisheries, Japan. The authors are grateful for the technical assistance of Yumiko Endoh of the National Institute of Livestock and Grassland Science, Japan. 


\section{References}

Aoyagi Y, Narumiya K, Itoh S, Sasaki K and Nakaya T. Effect of L-ascorbic acid-2-phosophate magnesium on chicks administrated a radical initiator (in Japanese). Japanese Poultry Science, 33: 383-387. 1996.

Aoyagi Y, Ohnishi T, Itoh S and Nakaya T. Effect of heat stress and L-ascorbic acid-2-phosphate magnesium on plasma and liver thiobarbituric acid reactive substances concentrations, and on liver protein carbonyl concentration in chicks (in Japanese). Japanese Poultry Science, 34: 63-66. 1997.

Haugan JA, Aakermannand T and Liaaen-Jensen S. Isolation of fucoxanthin and peridinin. Methods in Enzymology, 213: 231-245. 1992.

Mitsumoto M, Arnold RN, Shaefer DM and Cassens RG. Dietary versus postmortem supplementation of vitamin $\mathrm{E}$ on pigment and lipid stability in ground beef. Journal of Animal Science, 71: 1812-1816. 1993.

Mitsumoto M. Dietary delivery versus exogenous addition of antioxidants. In: Antioxidants in muscle foods (Decker EA, Faustman C and Lopez-Bote CJ eds.). pp. 315-343. WileyInterscience. New York. 2000.

Mortensen A and Skibsted LH. Antioxidant activity of carotenoids in muscle foods. In: Antioxidants in muscle foods (Decker EA, Faustman C and Lopez-Bote CJ eds.). pp. 6183. Wiley-Interscience. New York. 2000.

National Research Council. Nutrient requirements of poultry. Ninth revised edition. National Academic Press. Washington, D.C. 1994.

Niki E. Free radical initiators as source of water- or lipid-soluble peroxyl radicals. Method in Enzymology, 186: 100-108. 1990.

Nomura T, Kikuchi M, Kubodera A and Kawakami Y. Protondonative antioxidant activity of fucoxanthin with 1,1diphenyl-2-picrylhydrazyl (DPPH). Biochemistry and Molecular Biology Intternational, 42: 361-370. 1997.

Panda AK, Ramarao SV, Raju MVLN and Chatterjee RN.
Effect of dietary supplementation with vitamins $\mathrm{E}$ and $\mathrm{C}$ on production performance, immune responces and antioxidant status of White Leghorn layers under tropical summer conditions. British Poultry Science, 49: 592-599. 2008.

Sachindra NM, Sato E, Maeda H, Hosokawa M, Niwano Y, Kohno $\mathbf{M}$ and Miyashita K. Radical scavenging and singlet oxygen quenching activity of marine carotenoid fucoxanthin and its metabolites. Journal of Agricultural and Food Chemistry, 55: 8516-8522. 2007.

Sasaki K, Koga K, Narita R, Fukuda T, Kitaguchi Y and Aoyagi Y. Glutathione-dependent dehydroascorbate reductase activity in liver of chicken treated with dehydroascorbate or radical initiator (in Japanese). Japanese Poultry Science, 39: J41-J45. 2002b.

Sasaki K, Sano M, Satoh J, Ohishi Y, Itoh S, Nakaya T and Aoyagi Y. The effects of L-ascorbic-2-phosphate magnesium on chicks injected a radical initiator (in Japanese). Japanese Poultry Science, 39: 179-184. 2002a.

Sasaki K, Ishihara K, Oyamada C, Sato A, Fukushi A, Arakane T, Motoyama M, Yamazaki M and Mitsumoto M. Effects of fucoxanthin addition to ground chicken breast meat on lipid and colour stability during chilled storage, before and after cooking. Asian-Australasian Journal of Animal Sciences, 21: 1067-1072. 2008.

Sugawara T, Kushiro M, Zhang H, Nara E, Ono H and Nagao A. Lysophosphatidylcholine enhances carotenoid uptake from mixed micelles by Caco-2 human intestinal cells. Journal of Nutrition, 131: 2921-2927. 2001.

Sugawara T, Baskaran V, Tsuzuki W and Nagao A. Brown algae fucoxanthin is hydrolyzed to fucoxanthinol during absorption by Caco-2 human intestinal cells and mice. Journal of Nutrition, 132: 946-951. 2002.

Witte VC, Krause GF and Baily ME. A new extraction method for determining 2-thiobarbituric acid values of pork and beef during storage. Journal of Food Science, 35: 582-585. 1970. 